\title{
A novel access integrated system for electric power telecommunication
}

\author{
Kai Liang ${ }^{1, a}$, Fanbo Meng ${ }^{2}$, Yanping Zhang ${ }^{1}$, Jun Liu $^{1}$, Dan Yi $^{1}$, Xuexu You ${ }^{1}$, Yu Zou ${ }^{3}$ \\ ${ }^{1}$ State Grid Anshan Electric Power Supply Company, Anshan 114002, China \\ ${ }^{2}$ State Grid Liaoning Electric Power Company Limited, Shenyang 110006, China \\ ${ }^{3}$ Liaoning Planning and Designing Institute of Post and Telecommunication Company Limited, \\ Shenyang 110011, China \\ aamengfb@163.com
}

Keywords: Electric power telecommunication, OFDMA, sub-band division, metro-access integrated network.

\begin{abstract}
With the development of diversified communication services in smart grid, user demand and communication capacity have increased constantly, which imposes severe challenges on electric power telecommunication. Especially, the Metro Access Network (MAN) needs to aggregate and exchange a wider variety of services. However, metro and access networks developed independently, thus consuming a lot of network resources in complex infrastructures, which increases the development and operational costs of network operators. To address these problems above, we propose and demonstrate a novel metro-access integrated network based on Intensity Modulation and Direct Detection (IM/DD) Orthogonal Frequency-Division Multiple Access (OFDMA) techniques. It consists of a single fiber ring and many single fiber trees, and transparently integrates metropolitan area networks with optical access networks. The single fiber ring connects core networks and Central Nodes (CNs) which can allocate subcarriers according to user demand. Meanwhile, they can also implement flexible power distribution. The Remote Nodes (RNs) distributed on the user side are connected with the corresponding $\mathrm{CN}$ through the single fiber tree. Simulation results indicate that our proposed metro-access integrated network is feasible for electric power telecommunication while ensuring agile power distribution.
\end{abstract}

\section{Introduction}

With the increasing demand for emerging services in smart grid, the bandwidth requirement for access network is rising dramatically, leading to the increasing number of end users. Meanwhile, a large number of different communication methods need to be integrated and exchanged in metro networks. The access capacity will be soon put in a tight spot since the throughput capacity of metro networks is limited. Furthermore, the metro network and access network develop independently for current electric power telecommunication, which consumes a large amount of infrastructures and energy [1-3]. As a result, the integration of metro and access networks has gained great research interests. Especially, the long reach Passive Optical Network (PON) which is connected to the core network directly has become the best choice to meet this requirement. Besides, Orthogonal Frequency Division Multiplexing (OFDM) based PON has been widely studied since it is spectrum-efficient and flexible in bandwidth allocation [4].

The long reach PON has a ring-based topology, while the access part utilizes a tree-based topology. Thus, the existing long reach PON exhibits the characteristics of simple scalability, renewability and high bandwidth. There are two structures for metro-access integrated networks. The first one is an advanced scalable passive access network proposed by European researchers. In this structure, a passive add/drop remote node is used for the connection between Wavelength-Division-Multiplexing (WDM)-based two-fiber path ring and Time-Division-Multiplexing (TDM)-based single fiber tree. The other one is the metropolitan access ring integrated network. The two modes mentioned above share the infrastructures and information rate through TDM and WDM techniques. However, TDM requires strict time synchronization, while WDM needs expensive tunable lasers to satisfy the 
bandwidth demand of end users. Apparently, the aforementioned access methods are complicated or expensive. In contrast, the optical OFDM is a promising candidate solution for access networks since it has the advantage of high spectrum efficiency, strong tolerance of fiber dispersion and flexible bandwidth allocation [5].

In this paper, we propose a metro-access integrated system based on OFDM technique and demonstrate its feasibility successfully. In the proposed system, the single fiber ring is connected with a single fiber tree through Central Nodes (CNs) which achieves the communication routing. Moreover, the integrated network can improve system capacity and flexibility since new CNs can be set according to the user demand. The remainder of this paper is organized as follows. In section 2, we introduce the proposed metro-access integrated system. The simulation setup and results are shown in section 3. Finally, we conclude this paper in section 4.

\section{Metro-access integrated system for electric power telecommunication}

Generation of electrical OFDM signal. Based on the OFDM transmitter mentioned in [4], we introduce sub-band division in our system, which means that the sub-band carries information for different end users. Sub-band division is performed before Inverse Fast Fourier Transform (IFFT) module. Zero padding which is treated as guard-band between different sub-bands is executed between a certain number of data-carrying subcarriers. Figure 1 shows the multi-band OFDM signal spectrum.

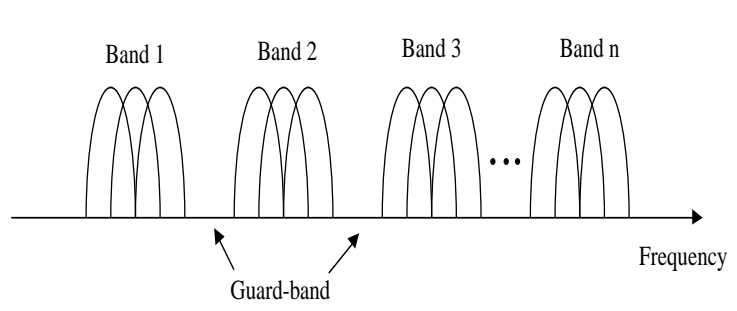

Figure 1 Schematic of multi-band OFDM signal spectrum

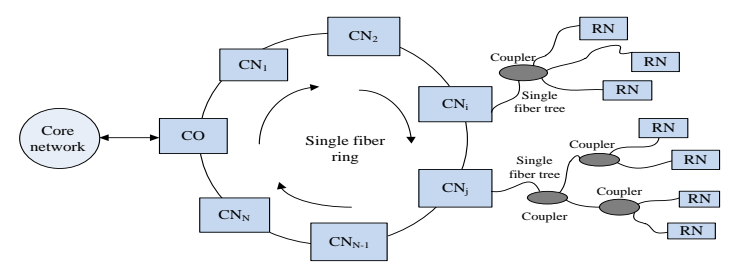

CO: Central Office CN: Central node RN: Remote node

Figure 2 Proposed metro-access integrated system based on OFDM technique

In each sub-band, the subcarriers are still adjacent orthogonal with each other, which means that the sub-band division does not influence the orthogonality between subcarriers. The number of subcarriers in each sub-band is arbitrary and can be adjusted dynamically according to the user demand.

Principle of metro access network integration. The proposed metro-access integrated network adopts the combination of ring and tree-based topologies and can allocate power and subcarrier dynamically suing OFDM technique. Furthermore, it connects with core network only through an Optical Line Terminal (OLT), which achieves the effective integration between metro and access networks beyond metropolitan area scope. As a result, the network integration degree can be improved and the network structure can be simplified.

Figure 2 demonstrates the proposed metro-access integrated system based on OFDM technique. It consists of a single fiber ring and multiple single fiber trees. Among which, the single fiber ring is constructed by a Central Office (CO) and several CNs. The CO connected with core networks is directly responsible for the entire network data aggregation and switch with the functions of resource control and allocation. There is an optical fiber link with the same direction between any $C N_{i}$ and $C N_{j}$, where $i=1,2 \ldots n, j=1,2 \ldots n-1$. Thus, each $\mathrm{CN}$ implements the data switch and routing independently. In this way, the CN can be added and deleted based on the demand of Remote Notes (RNs) without affecting other CNs, which can guarantee the normal operation of the whole network. RNs connect with the $\mathrm{CN}$ through a single fiber tree. It should be noted that the single fiber ring is bidirectional and the single fiber tree is unidirectional.

In the downstream direction, the data streams from core networks arrive at $\mathrm{CO}$ and are converted into the optical OFDM signal. It is a multiple band signal which consists of a different group of orthogonal subcarriers carrying diverse information. The sub-band distribution is actually the distribution of the user's signal. There are two methods for signal allocation. One is dynamic 
allocation where users can pick up any one or multiple bands according to their demand, which means that the variable rate access can be achieved. The other one is fixed allocation where only certain sub-band signals are transmitted to corresponding RNs.

After optical transmission, the downstream optical OFDM signal first enters into $C N_{1}$ where an optical amplifier is used to compensate for the transmission loss and attenuation. The signal is divided into two parts after the optical splitter, one part is continually transmitted into $\mathrm{CN}_{2}$ and another part is transmitted into the RNs connected with $C N_{1}$. RN receiver demodulates the received signal and transmits the signal to corresponding users.

In the upstream direction, the user data is first converted into the multiple band optical OFDM signal in RNs, and then the OFDM signal is transmitted into corresponding CNs through fiber links. Except the last CN, all CNs will transmit their received upstream optical OFDM signal to next CN. Finally, all upstream optical OFDM signals are aggregated at CO who will process all the upstream signals that will be transmitted to core networks.

\section{Simulation setup and results analysis}

Simulation setup. Three CNs $\left(C N_{1}, C N_{2}, C N_{3}\right)$ are employed in simulations, each of which owns an RN. The same transmitter and receiver are set in each CN and RN. In the CO transmitter, the total number of subcarriers is 256 and the number of useful subcarriers is 128 since Hermitian symmetry is utilized to generate a real-valued signal after IFFT. In this simulation, the whole OFDM frequency band is partitioned into three sub-bands, each of which consists of 32 subcarriers. 16 subcarriers are used for a guard band between two sub-bands. The Cyclic Prefix (CP) ratio is 1/8.

Then training symbols are inserted for symbol synchronization and equalization. The real-valued signal obtained from IFFT and its Hilbert transform are input into the two arms of Mach-Zehnder Modulator (MZM). After optical modulation, one part of the resulting signal is transmitted into $C N_{1}$ and another part is transmitted into $R N_{1}$. In the RN, the downstream optical OFDM signal is separated from the optical carrier. A Photodiode (PD) is used for converting the optical signal into an electric signal. Meanwhile, $R N_{1}$ transmitter also generates upstream optical OFDM signal in the same way as in $\mathrm{CO}$ and sends its signal to $C N_{1}$. The combined signals are sent to $C N_{2}$. The other CNs and RNs are the same as $R N_{1}$ and $C N_{1}$. The only difference is that $C N_{3}$ transmits upstream OFDM signal back to CO.

Simulation results and analysis. Figure 3 shows the diagram of double sideband spectrum for three sub-bands in $\mathrm{CO}$ transmitter. Then, the single sideband signal is acquired after optical modulation by using these three sub-bands signal and their Hilbert transform together to modulate MZM. The single sideband spectrum is depicted in Fig. 4.

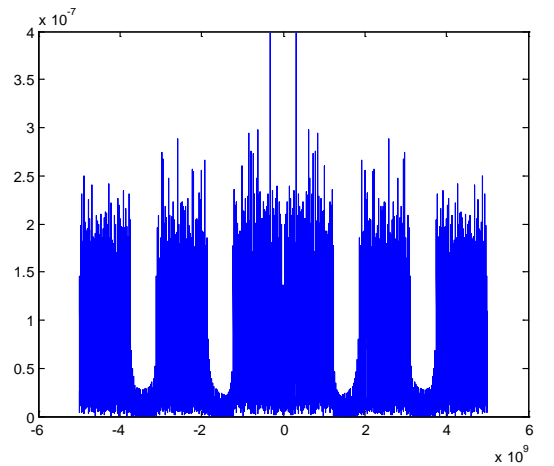

Figure 3 Diagram double sideband spectrum

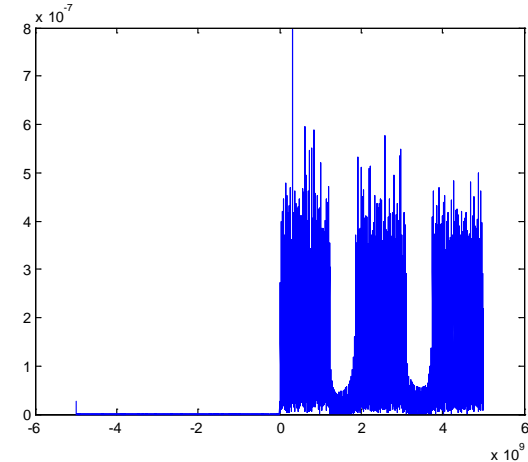

Figure 4 Diagram single sideband spectrum

Figure 5 demonstrates the BER performance as a function of for three sub-bands with the identical power allocation. We can see that three sub-bands have the similar BER performance. Next, the constellation and BER performance will be investigated under different ways of power allocation, in order to further verify the system performance in Fig. 6. We set the power allocation proportion among three sub-bands (sub-band1 (band1 in short), sub-band 2 (band-2 in short), sub-band3 (band3 in short)) is $8: 6: 1$, respectively. 
It can be seen that the constellation is clearer when the higher power is allocated, on the contrary, the constellation is messier when the lower power is allocated. Figure 7 displays the BER performance as a function of $\mathrm{E}_{\mathrm{b}} / \mathrm{N}_{0}$ for three sub-bands with different ways of power allocation. With the increasing $E_{b} / N_{0}$, the BER becomes better. As expected, when the $E_{b} / N_{0}$ is the same for three sub-bands, the sub-band with a higher power allocation exhibits a better BER performance.

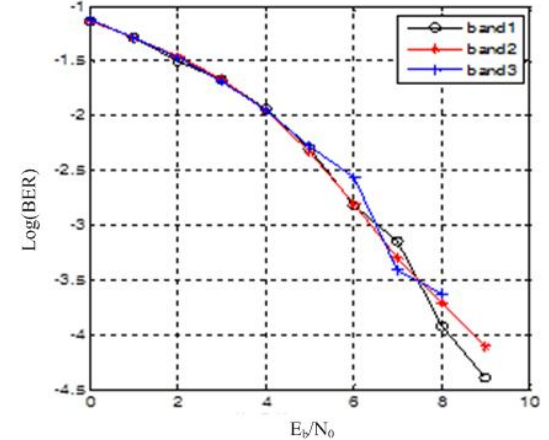

Figure 5 BER versus $E_{b} / N_{0}$ for three sub-bands with identical power allocation
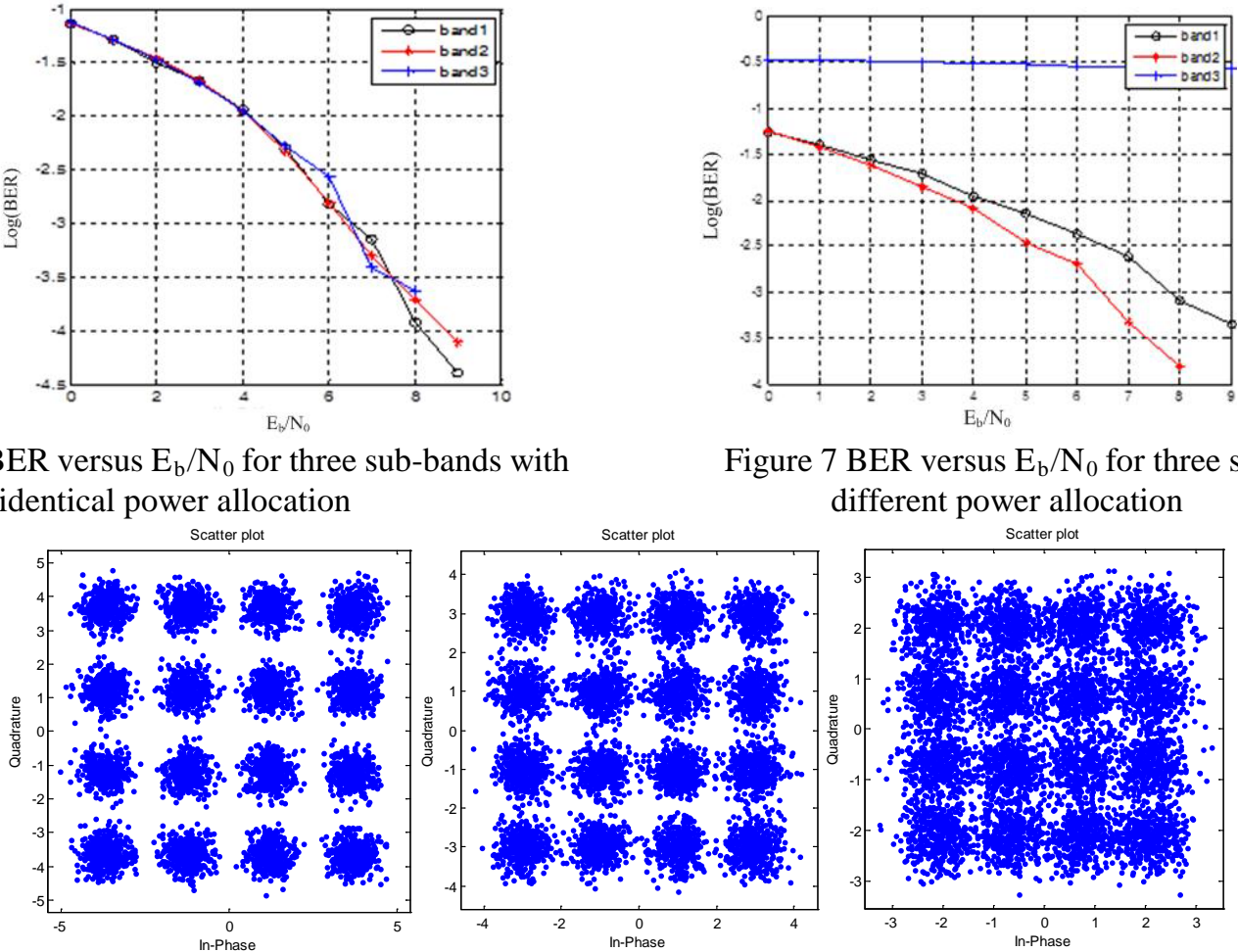

Figure 7 BER versus $\mathrm{E}_{\mathrm{b}} / \mathrm{N}_{0}$ for three sub-bands with different power allocation

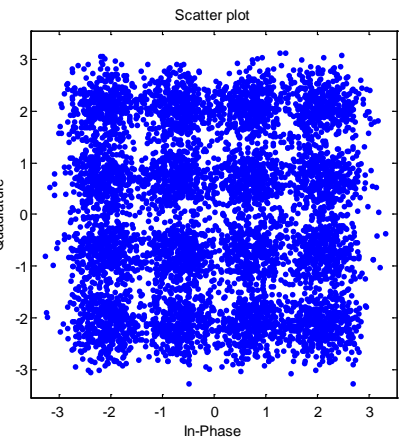

Figure 6 Constellations for three sub-bands after channel equalization

\section{Conclusion}

In this paper, we have proposed a novel metro-access integrated network for electric power telecommunication based on OFDM technique. By using the combination of the ring- and tree-based topologies, the metro and access networks can be integrated perfectly. Simulation results have shown that the proposed system is feasible and can be a promising candidate for electric power telecommunication.

\section{References}

[1] X. Gong, Lei Guo, Y. Liu, Y. Zhou, H. Li. Optimization mechanisms in multi-dimensional and flexible PONs: challenging issues and possible solutions, Optical Switching and Networking, 2015, vol. 18, no. Part1, pp. 120-134.

[2] Pedro E. D. Cruz, Tiago M. F. Alves, and Adolfo V. T. Cartaxo. Loss Budget of Ultra-Dense 10 Gb/s Per-User Guaranteed Direct-Detection MB-OFDM Metro-Access Network s, in Proc. ICTON, 2015, pp. Tu.B 1. 4.

[3] Pedro E. D. Cruz, Tiago M. F. Alves, and Adolfo V. T. Cartaxo. Impact of Inter-Band Crosstalk Due to Nonlinear Fiber Transmission on the Performance of Direct- Detection Single -Sideband MB-OFDM Metro Networks, in Proc. ICTON, 2015, pp. Tu.B 1. 3.

[4] W. Peng, B. Zhang, K. Feng, X. Wu, A. Willner, and S. Chi. Spectrally Efficient Direct-Detected OFDM Transmission Incorporating a Tunable Frequency Gap and an Iterative Detection Techniques, Journal of Lightwave Technology, 2009, vol. 27, no. 24, pp. 5723-5735. 
[5] X. Gong, L. Guo, J. Wu, and Z. Ning. Design and performance investigation of LDPC-coded upstream transmission systems in IM/DD OFDM-PONs, Optics Communications, 2016, vol. 380, no. 2016, pp. 154-160. 